\title{
A Non-Surgical Condition of Neonatal Pneumoperitoneum: Retroperitoneal Free Air Secondary to Massive Tension Pneumothorax
}

\author{
Unal Bakal ${ }^{\mathrm{a}}$, Mustafa Aydin ${ }^{\mathrm{b}, \mathrm{c}}$, Aysen Orman ${ }^{\mathrm{b}}$, Erdal Taskin ${ }^{\mathrm{b}}$, Ahmet Kazez $^{\mathrm{a}}$
}

\begin{abstract}
Pneumoperitoneum in newborns is usually an emergent surgical condition which indicates alimentary tract perforation and therefore threatens the life. However, there are some non-surgical conditions causing pneumoperitoneum which do not require the explorative laparotomy. This report describes a rare case of neonate with pneumoperitoneum developed secondary to massive tension pneumothorax. Resolution of the free air in the retroperitoneal space was observed after tube thoracostomy. This case report suggests that massive tension pneumothorax can be a rare cause of non-surgical pneumoperitoneum.
\end{abstract}

Keywords: Pneumoperitoneum; Pneumothorax; Newborn infant; Retroperitoneum; Laparotomy; Tube thoracostomy

\section{Introduction}

Neonatal pneumoperitoneum (NP), in general, is an acute abdominal surgical emergency which needs surgical intervention to ensure survival. It is most commonly caused by perforation of the gastrointestinal tract. However, there are some non-surgical conditions of the NP which have different etiologies and can be improved without explorative laparotomy $[1,2]$. Hence, NP can be categorized into two groups: surgical and non-surgical. Herein, we report a rare case of neonate with non-surgical condition of pneumoperitoneum developed secondary to massive tension pneumothorax.

Manuscript accepted for publication November 19, 2015

aDepartment of Pediatric Surgery, Firat University School of Medicine, Elazig, Turkey

${ }^{b}$ Department of Pediatrics-Neonatology, Firat University School of Medicine, Elazig, Turkey

${ }^{\mathrm{c} C}$ Corresponding Author: Mustafa Aydin, Department of Pediatrics-Neonatology, Firat University School of Medicine, 23119 Elazig, Turkey.

Email: dr1mustafa@hotmail.com

doi: http://dx.doi.org/10.14740/jmc2371w

\section{Case Report}

A female baby was born at 26 weeks' gestation by emergent cesarean section, due to fetal distress, to a 21 -year-old gravida 2 , para 1 mother. His birth weight was $640 \mathrm{~g}$. She was put on mechanical ventilation and then given surfactant because of the severe respiratory distress syndrome. While she was on mechanical ventilation on the 15 th postnatal day, a sudden desaturation and bradycardia occurred. On physical examination, she was cyanotic and bradycardic with an oxygen saturation of $55 \%$ and a heart rate of 90 beats/min, respectively. Auscultation of the chest demonstrated that right hemithorax did not

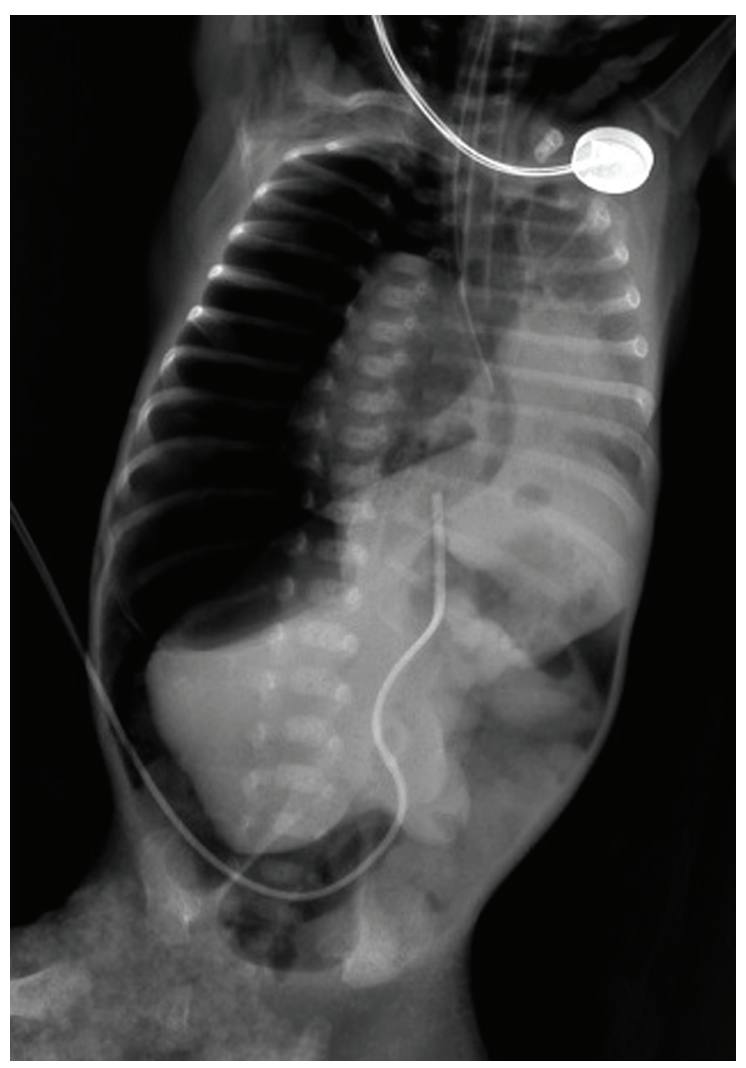

Figure 1. Thoraco-abdominal radiography demonstrating excessive amount of free air in the right-sided inter-pleural space which extended to the retroperitoneal area. 


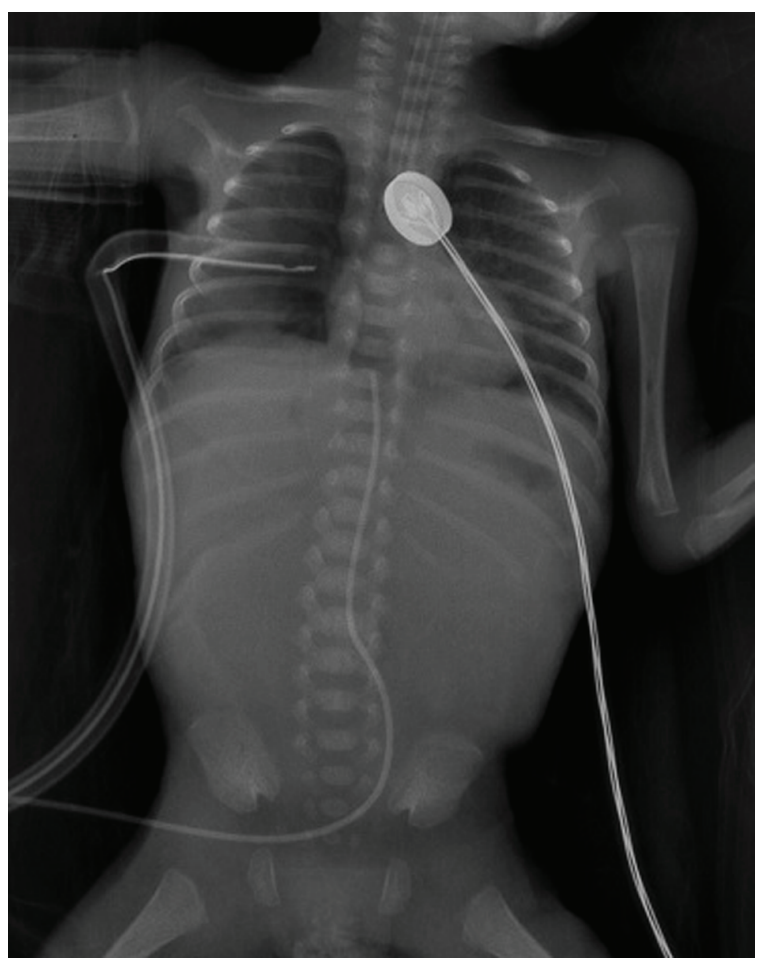

Figure 2. Control radiography showing resolution of the pneumoperitoneum after tube thoracostomy procedure.

participate in breathing well. The abdomen was also slightly distended without any sign of peritonitis. On laboratory, normal hemogram and C-reactive protein also excluded the abdominal surgical conditions. Emergent thoraco-abdominal radiography demonstrated excessive amount of free air in the right-sided inter-pleural space which extended to retroperitoneal area (Fig. 1). The diagnosis of pneumoperitoneum in this case was considered to develop secondary to massive tension pneumothorax by moving free air from inter-peritoneal space to retroperitoneum. After right-sided tube thoracostomy procedure, resolution of the free air in the retroperitoneum was observed on the control radiography (Fig. 2).

\section{Discussion}

Free gas under the diaphragm on abdominal radiography taken in an upright position usually suggests the diagnosis of gastrointestinal tract perforation [3]. However, primary diagnosis in our case was massive tension pneumothorax accompanying pneumoperitoneum. Furthermore, there were not any signs and symptoms associated with inflammation considering the diagnosis of peritonitis. Therefore, the diagnosis of alimentary tract perforation was doubtful in the present case. Also, improvement of pneumoperitoneum after management of pneumothorax has confirmed our idea.

In conclusion, pneumoperitoneum in newborns is usually an emergent surgical condition which indicates alimentary tract perforation and therefore threatens the life. But concurrent large amount of thoraco-abdominal free air, as observed in this case, should suggest a rare cause of non-surgical pneumoperitoneum secondary to massive tension pneumothorax. In addition, the present case demonstrates that in the cases of pneumoperitoneum without physical signs and laboratory findings of the generalized peritonitis, unnecessary laparotomy should be avoided.

\section{Conflicts of Interest}

The authors declare that they have no financial or non-financial conflicts of interest related to the subject matter or materials discussed in the manuscript.

\section{Grant}

None.

\section{References}

1. Gupta R, Bihari Sharma S, Golash P, Yadav R, Gandhi D. Pneumoperitoneum in the newborn: is surgical intervention always indicated? J Neonatal Surg. 2014;3(3):32.

2. He TZ, Xu C, Ji Y, Sun XY, Liu M. Idiopathic neonatal pneumoperitoneum with favorable outcome: A case report and review. World J Gastroenterol. 2015;21(20):64176421.

3. Aydin M, Zenciroglu A, Hakan N, Erdogan D, Okumus N, Ipek MS. Gastric perforation in an extremely low birth weight infant recovered with percutaneous peritoneal drainage. Turk J Pediatr. 2011;53(4):467-470. 